\title{
Actualización de un debate patrimonial a través del paisaje histórico y la creatividad urbana: preexistencias y ciudad histórica contemporánea
}

Plácido González Martínez | Dpto. de Historia, Teoría y Composición Arquitectónicas, Universidad de Sevilla

URL de la contribución <www.iaph.es/revistaph/index.php/revistaph/article/view/3589>

El conflicto existente entre las preexistencias y la ciudad contemporánea tiene una base cultural arraigada en Occidente. En virtud del entendimiento lineal del tiempo, todo lo que nos antecede recibe un valor de antigüedad que se encuentra en los orígenes del interés de la cultura europea por su patrimonio. Los primeros pasos que se dieron ya a mediados del siglo XVIII hacia esa valoración se produjeron en el momento en que el desarrollo tecnológico impulsó el avance del positivismo. Con él llegaron dinámicas de renovación constante y la asunción incondicional de una cultura de la obsolescencia, que difumina el trazo de esa línea temporal al dibujarse en la ciudad.

Los intentos por resolver ese conflicto, menos acusado en otras culturas en las que no se considera la linealidad del tiempo, ofrecen como resultado frecuentes paradojas. Una de ellas se presentaría a quien entendiera la historia como proceso orgánico: según esta interpretación, todas las intervenciones que se produjesen sobre la ciudad histórica terminarían, por selección natural, bien siendo integradas, bien rechazadas, generando una nueva sustancia urbana que se actualizara sin cesar. Se trataría de un enfoque fundamentable científicamente, con tantos datos y casos como quisiéramos. Pero de ser así, desde las intervenciones operadas por Sixto $V$ en Roma hasta las mutaciones acontecidas en el Pekín contemporáneo, podríamos explicarnos la transformación de las ciudades con la misma tranquilidad que la forma de las nubes: sin poder operar sobre ellas, solamente reconocerlas cuando acontecen.

\section{Patrimonio, vivencia y poder}

Nos recuerda Maurice Halbwachs que esa concepción orgánica de la historia, como estructura separada del espacio de la vivencia, puede ofrecer un bálsamo para la asimila- ción de los conflictos, pero no es suficiente para resolverlos (HALBWACHS, 1995). Un camino paralelo al de la historia fue el de la memoria, recorrido de manera natural como parte de la experiencia, que fue señalada para entender a las ciudades como escenarios de un proceso vital en movimiento continuo, desde la percepción del individuo.

La memoria sirvió de fundamento a aquellos que, a medida que el desarrollo industrial justificaba la destrucción de las ciudades históricas en Occidente, adoptaron una postura de resistencia frente al cambio. Postura que podía expresarse con una infinidad de matices, si bien para la posterior formulación del patrimonio quedaron los hitos de la oposición completa a la manifestación insensible del progreso, defendida por quienes encontraron motivación en el sentimiento de pérdida asociado a la melancolía -tal era el caso de John Ruskin- hasta el reformismo pragmático que dio pie a la exacerbación fantástica de la nostalgia, como bien pudieron ilustrar las obras y escritos de Viollet Le Duc.

En el marasmo de los cambios, durante el siglo XIX y buena parte del siglo XX, la cultura de la modernidad dispuso de la preexistencias de manera completamente desprejuiciada, en cualesquiera de las esferas de la ciencia, la moralidad y el arte que definió Weber. Trasladado a la ciudad, este discurso se planteó en términos de pura yuxtaposición, y fueron raras las ocasiones en las que se reflexionó sobre los principios que subyacían en esa conflictiva mezcla de lo preexistente y lo contemporáneo. Las reformas del Barón Haussman en París a lo largo de la segunda mitad del siglo XIX, o las operaciones de apertura de las "grandes vías" en capitales españolas como Madrid, Sevilla o Granada a inicios del siglo $\mathrm{XX}$, pueden dar buena muestra de ello ${ }^{1}$. 
Para las disciplinas de la arquitectura y el urbanismo, se obvió el debate sobre la naturaleza diversa de los poderes que se habían manifestado a lo largo de siglos en la ciudad histórica, y los que confluían para generar la ciudad contemporánea, dejando lagunas de incomprensión que siguen sin sondearse hoy día. La cuestión estética se desplegó como una cortina de humo, escamoteando la posibilidad de influir en esas dinámicas de cambio más allá que en la decisión sobre los ropajes con los que vestir a la arquitectura. Volviendo a las "grandes vías", el caso de Madrid es ejemplar con el rascacielos de Telefónica, proyectado por Ignacio de Cárdenas entre 1926 y 1929 , que tras su fachada neobarroca ocultaba sistemas estructurales y de instalaciones de auténtica vanguardia en su generación.

Incorporándose este sesgo artístico como complemento al temporal, la producción de nueva arquitectura en las ciudades históricas buscó frecuentemente justificación en disquisiciones sobre la analogía y el contraste. Posturas que de nuevo hablan de extremos, de difícil concreción, máxime cuando esos extremos ocupan posiciones variables, y entre ellos son múltiples los recorridos posibles. Sin embargo, todas las reflexiones que pudieran hacerse sobre los estilos, siendo útiles, prolíficas y de interés, no alcanzaban a reconocer la complejidad de los fenómenos que se manifestaban en este escenario.

\section{La ciudad de la disciplina}

Siendo el estético un capítulo importante, pero en absoluto decisivo, de esta problemática, nuevos factores se incorporaron al debate en Europa tras la Segunda Guerra Mundial. La experiencia traumática de los conflictos que asolaron el continente durante la primera mitad del siglo XX motivó un cambio de actitud frente a la ciudad histórica, cuyas consecuencias siguen aún vigentes. El "corazón" de la ciudad, como se le llamó en el 8. ${ }^{\circ}$ Congreso Internacional de Arquitectura Moderna (CIAM) de 1951, reclamó un nuevo protagonismo en la reconstrucción física y espiritual del continente desde una perspectiva humanista que bebía de las fuentes de Heidegger, favoreciendo una atención renovada al pasado y un cambio sensible en la modernidad arquitectónica europea.

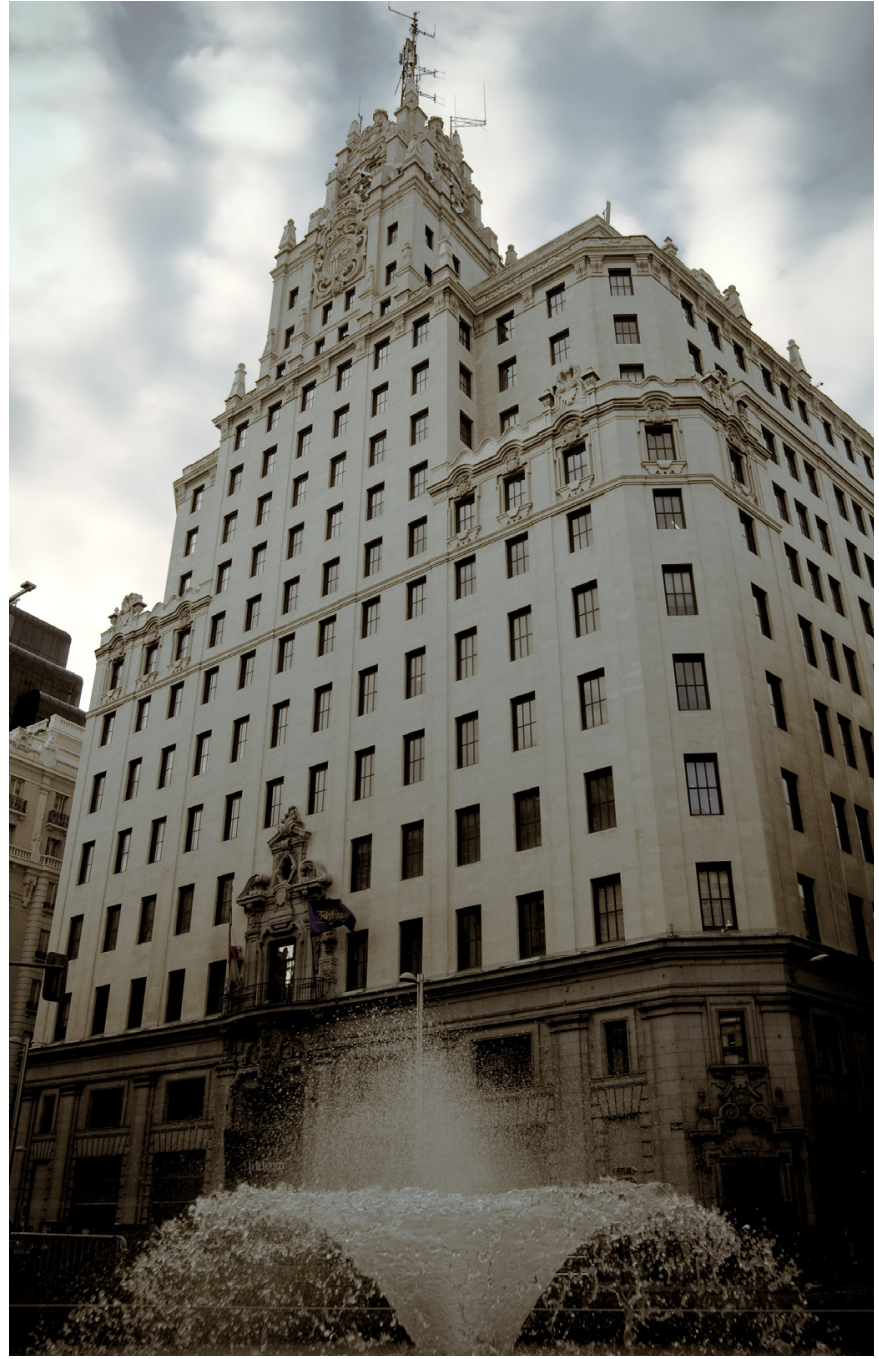

Edificio de Telefónica en la Gran Vía, Madrid (arquitecto: Ignacio de Cárdenas, 1926-1929) | foto Andrés López (http://www.redbubble.com/people/alopezm/; http://www.antoniolopezmurillo.tk/)

Las respuestas, se consideró entonces, debían traspasar la membrana impermeable de lo epidérmico, buscando el código genético de la ciudad, que permitiese entroncar la producción de arquitectura contemporánea con los tejidos urbanos preexistentes. La reflexión teórica que tuvo lugar en Italia durante los años 50 y 60, alimentada por Saverio Muratori y continuada, entre otros, por Gianfranco Caniggia², ofreció una nueva manera de entender las relaciones entre la ciudad histórica y una 


\section{a debate Preexistencias en la ciudad histórica contemporánea: viabilidad y convivencia}

| coordinan Plácido González Martínez, Miguel Ángel Tabales Rodríguez

condición contemporánea ya abocada a la exacerbación de la cultura frente a la técnica; efecto secundario, que no menor, de la globalización.

A partir del despertar de esta sensibilidad de la mano de lo que se llamó la "historia operativa", arquitectos como Aldo Rossi y otros miembros destacados del movimiento de la Tendenza, vinculados todos ellos a la izquierda política, sentaron las bases para un abordaje disciplinar desde la teoría de la arquitectura al diálogo entre lo preexistente y lo contemporáneo (ROSSI, 1999). Éste fue el principio de un conservacionismo progresista, tan paradójico en su denominación como consecuente a nivel conceptual, que se fundamentó en dos cuestiones fundamentales:

> El establecimiento de una "ciencia urbana" gobernada por principios y parámetros exclusivamente arquitectónicos, y fundamentada en los principios del análisis urbano estructuralista;

> La consideración hacia la cuestión de la identidad, que atendiese a la continuidad de la presencia de ciertos grupos sociales en la ciudad histórica como garantía de conservación.

Ambos aspectos fueron decisivos para caracterizar el abordaje que se dio en llamar "culturalista" a la ciudad histórica contemporánea ${ }^{3}$, y tuvieron los casos de Bolonia y Brescia como ejemplos destacados de los logros que la atención a la morfología urbana y a las tipologías edificatorias podía procurar. Gracias a las intervenciones de Pierluigi Cervellatti y Leonardo Belevolo respectivamente, los centros históricos de ambas ciudades fueron capaces de acometer los cambios que precisaron para su revitalización, sirviendo de referentes para los principios inclusivos que la Declaración de Ámsterdam consagraría más tarde en 1975.

Esta apuesta por la conservación, también conocida como rehabilitación integral, tuvo autoridad a nivel disciplinar aunque una repercusión limitada en las intervenciones en el resto de las ciudades históricas europeas a lo largo de los años 60 y 70, si bien cobró plena actualidad con la crisis económica de 1973. La constatación de los límites del crecimiento fue un acicate para la economización de recursos, al mismo tiempo que evidenció ciertos riesgos que podían cernirse sobre los centros históricos si la arquitectura y el urbanismo se entendían exclusivamente como ciencias provistas de sus propios procedimientos normativos.

Bolonia ofreció también un buen ejemplo de esta problemática, puesto que en ella se dieron la mano dos tendencias de máxima contemporaneidad que afectaban de manera distinta a centro y periferia. Por un lado, en la ciudad histórica, el culturalismo conservacionista propició la intensificación de su carácter y su actualización funcional manteniendo su composición social, si bien a una velocidad poco acompasada con los cambios que operaban en la economía.

Mientras, en la periferia norte de la ciudad, la velocidad era otra, como se evidenció en uno de los contados desembarcos de la arquitectura metabolista en Europa, de la mano del arquitecto japonés Kenzo Tange, con el desarrollo del descomunal complejo de la Feria de Bolonia, siguiendo la moda de los "Centros direccionales", tan en boga en Italia durante la década de los 60 y 70.

La dialéctica entre centro y periferia, denunciada en su momento por Henri Lefebvre, se desvela contemplando Bolonia a vista de pájaro, y comparando la escala de su centro y la Feria de Tange ${ }^{4}$. Sirve para dar cuenta de la magnitud de los cambios que acontecían alrededor de la ciudad histórica, permitiendo la implantación del discurso de las megaestructuras en el territorio cercano de manera completamente autónoma. Noli me tangere, el blindaje de la ciudad histórica podía provocar el efecto secundario de una existencia autista, insensible a las actuaciones que pudieran emprender unos poderes públicos entregados a la acción de los promotores.

\section{La ciudad histórica, nuevas dinámicas de cambio y nuevas respuestas}

El hiperdesarrollo suburbano terminó provocando la 




Vista de Bolonia, Italia | foto Lorenzo Click (https://www.flickr.com/photos/ lorenzoclick/)

obsolescencia del corazón de la ciudad a partir de los años 60. Este proceso, contestado en los Estados Unidos de posguerra por Jane Jacobs, se manifestó con fuerza en las ciudades europeas de manera casi simultánea, y sus efectos se prolongaron hasta bien entrada la década de los 80. En la ciudad histórica, los más evidentes fueron el abandono y el decaimiento de su tejido, y en un marco de globalización económica generalizada, las respuestas que pudieran dar la visión disciplinar -con su rigor metodológico estructuralista- o la posthistórica -con su fantasía compositiva postmoderna- de la arquitectura resultaban del todo insuficientes.

Tan dados a las metáforas epatantes, los estudios urbanos en los Estados Unidos popularizaron la denominación del "efecto donut", para describir el vaciamiento progresivo de las áreas centrales de la ciudad frente a la pujanza de la periferia. Y con no menos ingenio, pusieron también nombre al principal riesgo al que se enfrentaban unas ciudades históricas noqueadas, completamente desprotegidas frente a la capacidad de acción ilimitada que gozaba el capitalismo neoliberal: el efecto "donut relleno", que refería a la costumbre estadounidense de completar el hueco del famoso postre con cremas y siropes de dulcísimo sabor.

Ese relleno, traducido en términos urbanos, tenía como principales ingredientes la fachadización y la gentrifica-

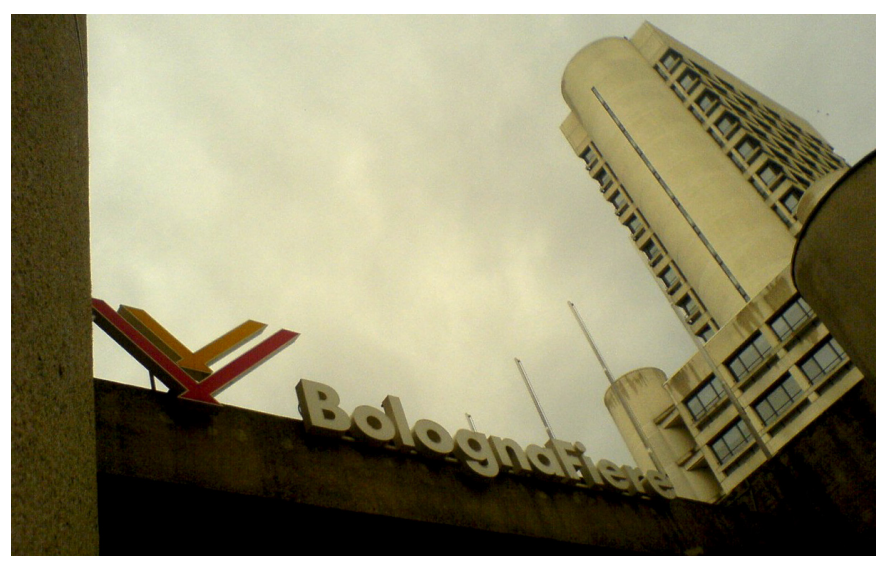

Sector de la Feria de Bolonia, Italia (arquitecto: Kenzo Tange, 1972) | foto Michele Mazzoli (https://www.flickr.com/photos/foread/)

ción. La Declaración de Ámsterdam de 1975, que vino a consagrar la ejemplaridad de experiencias como Bolonia y Brescia, evidenció todas sus limitaciones a la luz de las nuevas políticas económicas, que en la Europa meridional ofrecieron al turismo y al sector inmobiliario la nueva responsabilidad motriz que anteriormente habían tenido la industria y la agricultura. Este "endulzamiento", que con demasiada frecuencia llevó a la banalización de las preexistencias en la ciudad histórica contemporánea, estuvo motivado por la implementación de mecanismos de gestión público-privada, que se convirtieron en hegemónicos en los años de euforia previos a la actual crisis económica, entre 1996 y 2008.

Pero al igual que ocurrió en los años previos a la Crisis del Petróleo en los años 70, en ese impasse se formuló la contestación a las derivas neoliberales descritas, y que ha cobrado plena actualidad una vez que se produjo en 2008 el estallido de la burbuja financiera: siguiendo el símil alimenticio, no hablaríamos ya de elegir el sabor del dulce, sino de un principio más modesto y contingente como el de no morder la mano que da de comer.

En el marco temporal lineal que describíamos como contexto, nos encontramos con la paradoja de que también las estrategias de resistencia que en su día diseñaron los arquitectos de la Tendenza devinieron obsoletas, y precisaron de actualización. 
a debate Preexistencias en la ciudad histórica contemporánea: viabilidad y convivencia

| coordinan Plácido González Martínez, Miguel Ángel Tabales Rodríguez

Reconociendo la validez de muchos de sus preceptos, nuestra tesis es plantear su continuidad como nuevas respuestas, mediante un reconocimiento de la multiplicidad de factores.

Fundamentalmente, habremos de tener en cuenta aquellos vinculados a las nuevas condiciones de la globalización económica y al impacto de las tecnologías de la información, lo que conlleva:

$>$ Ante las limitaciones de los planteamientos de una "ciencia urbana" basada en parámetros exclusivamente arquitectónicos, explotar las posibilidades de análisis complejo e intervención estratégica ligadas al nuevo paradigma del paisaje histórico urbano ${ }^{5}$.

$>$ Frente a la necesaria trascendencia económica de la cuestión de la identidad, implicar a expertos y residentes en la ciudad histórica en la definición del paradigma de la creatividad urbana, como táctica de conservación y revitalización del patrimonio cultural ${ }^{6}$.

Paisaje histórico urbano y creatividad urbana se ofrecen como nuevo marco metodológico de intervención al servicio de un proyecto definido de ciudad histórica, con relación al cual se establezcan principios de diálogo ente las preexistencias y la contemporaneidad. En ambos, el patrimonio converge con otros campos y disciplinas, obligándose a un esfuerzo de actualización que necesariamente supondrá su redefinición.

\section{Preexistencias, nuevos agentes y nuevos valores}

Tomando como válida la premisa estructuralista de la ciudad como texto, una consideración exclusivamente disciplinar dejaba la integración de la arquitectura contemporánea como competencia exclusiva del arquitecto como "autor". Los avances en la filosofía política, la filosofía de la naturaleza, y de los propios medios de comunicación, sitúan hoy día esa tarea en manos de "autores" mucho más diversos, cuya responsabilidad en la construcción de sentido es análoga a la que puedan tener los ciudadanos y los habitantes como tradicionales "lectores" de la ciudad.
Ese "texto", según la lógica postestructuralista, estaría hoy dividido en múltiples relatos, que constituyen una base operativa más inclusiva para abordar tanto los problemas de la intervención en la ciudad histórica, como la integración de la misma en el marco más amplio del territorio. Estos relatos contemplan actividades socioeconómicas y procesos históricos en los cuales las preexistencias y lo contemporáneo se mezclan, cobrando nuevo sentido en su lectura conjunta. Ya no se trata, frente al pasado, de la mera justificación estilística, morfológica o tipológica, que legitimaba por alusiones, sino de una proyección hacia el lugar y el tiempo presentes, apuntando a su desarrollo futuro.

El punto de partida es la identificación de aquellos agentes que contribuyen a la construcción del paisaje urbano, incidiendo en su capacidad de acrecentar o menoscabar los valores que se le asocian. En el nuevo parlamento productor de estos relatos, se identifican:

$>$ Los llamados agentes humanos, a quienes la UNESCO reconoce como cualificados en el punto 25 de la Recomendación sobre el Paisaje Histórico Urbano, contando con la ciudadanía, como productora; las instancias decisorias y políticas, como impulsoras, y los técnicos, profesionales y administradores, como gestores.

> Los denominados agentes no humanos (UNESCO, punto 19) de la misma recomendación, y a los cuales es preciso conferir además un papel activo, tales son el territorio, como expresión del escenario físico del paisaje, además de la flora y la fauna, que constituyen el complemento a la acción humana.

La definición de nuevos valores patrimoniales resulta crucial para establecer las condiciones de ese diálogo entre preexistencias y ciudad contemporánea, que nuestra cultura democrática exige que se desarrolle en el respeto profundo a las diferencias. Estos valores incorporarán lo relativo a la arquitectura y el urbanismo, junto a la historia, la cultura, la economía y la sociedad, permitiendo maximizar los puntos de contacto entre el patrimonio y la compleja realidad con que se pretende operar. 
Las experiencias desarrolladas por la Tendenza siguen teniendo una validez reconocida, y la visión operativa de la historia sigue ofreciendo un papel relevante a la estética, la morfología y la tipología. Esto requiere, al mismo tiempo, desembarazarse de las analogías textuales respecto a la ciudad histórica, puesto que mantienen al discurso arquitectónico anclado en opciones conservadoras, reivindicando un rol demiúrgico para los arquitectos que cuando menos es cuestionable: por supuesto, su intervención seguirá siendo completamente necesaria, aunque siempre que su aportación se incluya en marcos de referencia más amplios y enriquecedores, como los definidos por el Memorándum de Viena de 2005².

La visión de la ciudad histórica como palimpsesto otorga la carta de naturaleza para la arquitectura contemporánea mediante el cumplimiento de los requisitos de la autenticidad y la integridad, que el urbanismo moderno establece a través de planes y ordenanzas, y que requieren de una interpretación necesariamente generosa y abierta a la producción de alternativas. La autenticidad penalizará la comisión de falsos históricos, toda vez que buscan en el pasado la legitimación necesaria para el presente, si bien un discurso contemporáneo ha de avanzar sobre parámetros puramente estilísticos.

Una nueva sensibilidad incluirá también los relativos a la materialidad y la técnica, así como todos aquellos que definan a la ciudad histórica como un conjunto delicado de relaciones y equilibrios. Buen ejemplo de ello puede ofrecer la intervención del arquitecto portugués Álvaro Siza en la reconstrucción del barrio lisboeta del Chiado, ortodoxa en el respeto a los principios de la Tendenza, y cuidadosa a la hora de dar continuidad al tejido histórico a través de la arquitectura contemporánea, así como en la integración de las infraestructuras modernas de acuerdo al carácter de la ciudad.

De igual manera, la defensa de la integridad en las ciudades históricas apuesta decididamente por el mantenimiento de estructuras tradicionales de parcelario, sobre las que se puedan entender la sucesión natural de la arquitectura y la memoria de la génesis de la ciudad.

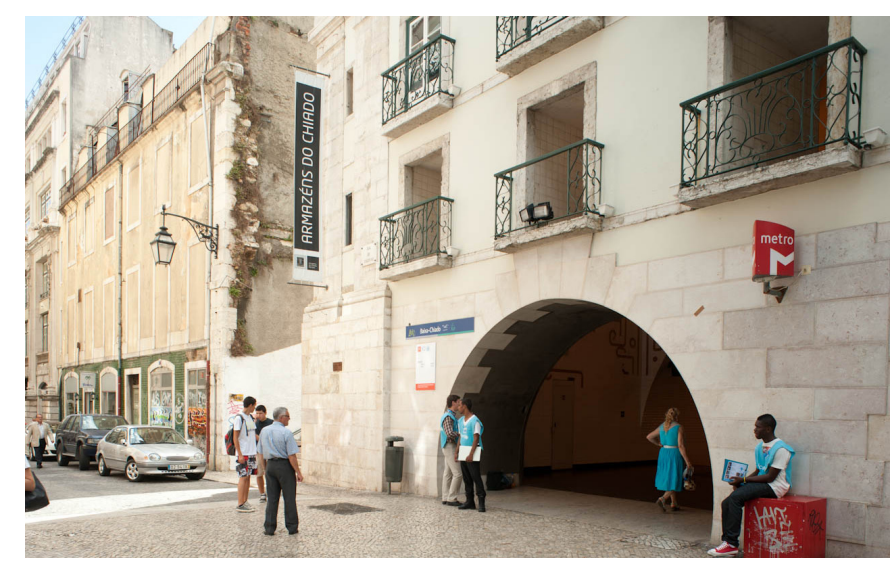

Acceso a la estación de Baixa-Chiado en Lisboa, Portugal (arquitecto: Álvaro Siza Vieira, 1992-1998) | foto Leon (https://www.flickr.com/photos/leonl/)

La quiebra de este principio suele venir de la mano de la avaricia del mercado inmobiliario, como se demostró en el Berlín de los años 90, cuando la estructura de propiedad estatal del suelo mantenida durante más de cuatro décadas en la República Democrática de Alemania fue traducida a los particulares y engañosos códigos del capitalismo neoliberal a partir de la reunificación del país.

Este fenómeno se agravó en la reconstrucción del barrio histórico de la Fiedrichstadt. Promovida por grandes corporaciones que favorecieron la creación de grandes parcelas, el cambio rompió así el equilibrio de un tejido urbano generado desde finales del siglo XVII, por más que la nueva arquitectura procurara atenuarlo con muestras de un fachadismo banal. Se enterró así el espíritu que anteriormente, con ocasión de la IBA de 1984, había procurado la "Renovación urbana respetuosa" en otros barrios centrales de la misma ciudad, tal fue el caso de Kreuzberg, y que hundía sus raíces en las experiencias italianas de los años $70^{8}$.

La cultura es parte fundamental de los valores a ser integrados dentro de esta visión. Las ciudades históricas se han convertido en depositarias de un acusado valor simbólico, frecuentemente enriquecido con la implantación de instituciones culturales de primer nivel: Bolonia ya mostró la vía en los años 60 , al programar la conversión 
a debate Preexistencias en la ciudad histórica contemporánea: viabilidad y convivencia

| coordinan Plácido González Martínez, Miguel Ángel Tabales Rodríguez

ordenada en museos y bibliotecas de numerosos edificios históricos. Hoy día, esta tendencia salta desde los edificios a los espacios públicos, con la creación de ejes culturales en los que se fomente la ralentización y disfrute ocioso de servicios, tal como defienden movimientos como la cittá slow.

Buen ejemplo de esa corriente puede ofrecer las crecientes tendencias a la peatonalización de entornos históricos, en las que es necesario considerar la trascendencia de la aportación de urbanistas como Jan Gehl. Su trabajo continuado desde finales de los años 60 cuenta con el hito de la propuesta de creación en 2008 de nuevos espacios públicos a lo largo de la avenida Broadway en Nueva York, que empezará a completarse en 2015 con el nuevo diseño del entorno de la mediática y patrimonializada Times Square.

La atención creciente a la ecología urbana ha reforzado la lectura territorial de la ciudad, potenciando la presencia de vías de agua, ejes pecuarios, vegetación o fauna, que para el urbanismo de los años 60 intentó corregir recurriendo a las infraestructuras.

Ejemplo de ello ofrecen las iniciativas puestas en marcha desde 2002 en la ciudad de Lyon, y que han llevado a la completa reconfiguración de las márgenes del río Ródano. La ambiciosa intervención de la paisajista Françoise Helène Jourda ha vuelto a conectar al centro histórico de la ciudad con el territorio a través de un corredor natural que se extiende a lo largo de cinco kilómetros, y ha sido aplaudida a nivel internacional por su calidad.

No menos interés despiertan las actuaciones que desde 2011 se vienen desarrollando en las márgenes del otro río de la ciudad, la Saône, que junto a la compleja consideración que ofrece a la naturaleza, incorpora elementos patrimoniales del curso tradicional del río y el centro histórico, acrecentados por una cuidada selección de intervenciones artísticas contemporáneas ${ }^{9}$.

En esta atención a la naturaleza, se incluyen también aquellos espacios urbanos no planificados en los que se hace patente la presencia de la naturaleza a partir de la obsolescencia y el abandono, tal y como ha descrito el paisajista francés Gilles Clément (CLÉMENT, 2005): ése fue el motivo para que se promoviera la recuperación del ferrocarril elevado que bordeaba el borde oeste de la isla de Manhattan y la creación del High Line por parte de los arquitectos Diller Scofidio y Renfro junto al paisajista Piet Oudolf, auténtico hito de las intervenciones paisajísticas en contextos urbanos consolidados de inicios del siglo XXI.

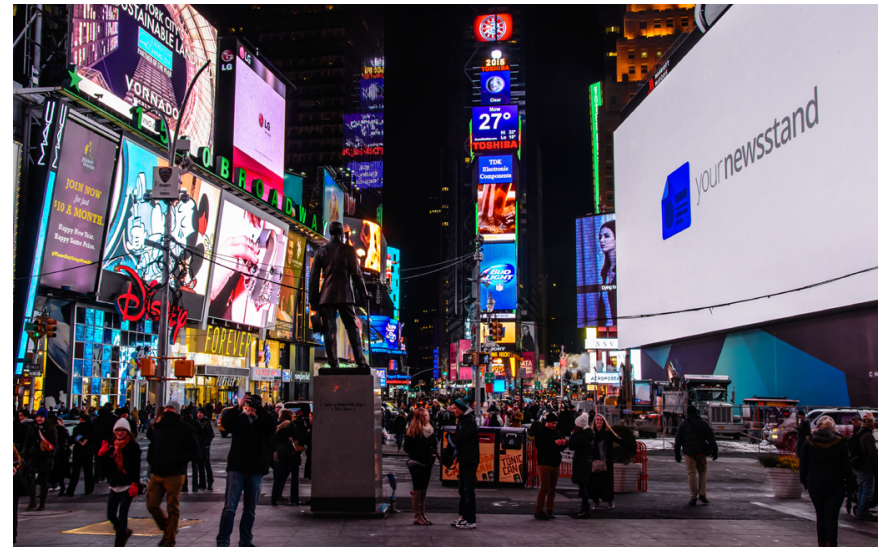

Reurbanización de Times Square, Nueva York, EEUU (arquitectos: Snohetta, 2010-2015) | foto drpavloff (https://www.flickr.com/photos/niceimages/)

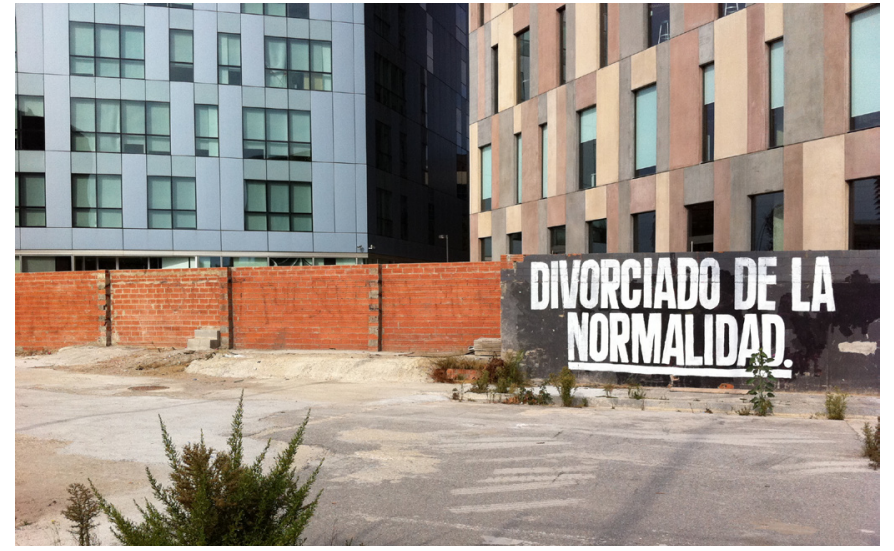

Sustitución tipológica en curso. Distrito 22@, Barcelona, España, 2011. | foto Plácido González Martínez 


\section{Los retos de la contemporaneidad}

Varios son los retos prioritarios para el futuro. El primero de ellos surge de reconocer la dimensión económica subyacente en el patrimonio cuando se expresa en términos de conservación energética. Siendo la adecuación a nuevos sistemas de producción de energía una cuestión aún irresuelta para la propia arquitectura contemporánea, ¿qué cabe esperar de su integración en la arquitectura histórica? Estudios recientes han incidido en la atención a esta cuestión, vinculada a la calidad del habitar y al fomento de técnicas tradicionales de construcción: experiencias recientes como las puestas en marcha por el Consorcio de Santiago de Compostela muestran posibles vías a seguir en este sentido (PANERO PARDO, 2011; ESTÉVEZ, 2004).

La generación de empleo, a la que acertadamente apunta el Consorcio de Santiago, es un aspecto especialmente importante dentro de la dimensión económica de la ciudad histórica, que en los últimos tiempos apunta al auge de la economía creativa, como ha sido definida por teóricos como Richard Florida ${ }^{10}$. En ella, se reconoce la relevancia de la ciudad histórica a la hora de atraer y fomentar las tres T, de Talento, Tecnología y Tolerancia.

Además de su evidente impacto en las industrias culturales, la manifestación de este nuevo protagonismo de las Ilamadas "clases creativas" supone también la intensificación de viejos y conocidos efectos secundarios, como la gentrificación. Buena prueba de ello dio la transformación del distrito 22@ en el Poblenou de Barcelona durante los últimos 15 años, y se repite actualmente con intensa repercusión mediática en el barrio histórico de The Mission en San Francisco, tras las actuaciones llevadas a cabo a lo largo de 2014 por los llamados Google Bus Protesters.

Aquí se revelan también las mayores esperanzas que puede encontrar el patrimonio en la ciudad histórica contemporánea: definir un nuevo paradigma de creatividad urbana en el que se incluya a una población empoderada. Esto motiva el fomento de la accesibilidad, para que los centros históricos sigan manteniendo una com- posición social variada y mixta, tanto étnica, de género como de nivel social y económico. Y se plantea como una cuestión necesaria que además de a las preexistencias, las acciones se dirijan hacia la mejora de las condiciones de vida de la gente, fomentando su corresponsabilidad en el mantenimiento y potenciación del patrimonio, facilitando la práctica de los principios democráticos y el ejercicio de los derechos fundamentales ${ }^{11}$.

Las iniciativas que se emprenden en esta dirección abandonan las premisas totalizadoras de la rehabilitación integral defendida por la Tendenza, y adoptan los modos más imperceptibles, de pequeña escala, de los que con frecuencia ha hecho uso el liberalismo económico a la hora de renovar la ciudad preexistente. En la medida en que el paradigma de la creatividad urbana se vincule a una idea general sobre la ciudad histórica compartida por todos los agentes que en ella participan, pretende desarrollar actuaciones intensas con pocos recursos, para aportar positivamente a la regeneración urbana en general, y de los espacios públicos en tejidos históricos en particular.

En esta línea, podríamos señalar, en primer lugar, la acción sobre la gestión, y la experiencias exitosas a nivel internacional desarrolladas con las herramientas de los presupuestos participativos, que permitieron la recuperación del emblemático Mercado Central de Porto Alegre en la segunda mitad de los años 90, o la recuperación del histórico barrio de la Garbatella en Roma, de la mano de la Associazione Culturale Controchiave. En segundo lugar, el ejercicio práctico que desarrollan colectivos multidisciplinares como Street Plans Colaborative en Miami, City Mine(d) en Londres y Bruselas, Ecosistema Urbano en Madrid, o Exyzt en París, que promueven la regeneración de edificios y espacios públicos amenazados por la obsolescencia, con un alcance cada vez más global.

Su apuesta por metodologías de actuación participativa marca un camino de gran potencial, multiplicando los afectos entre la población y su entorno construido como principio fundamental del hecho patrimonial. Si bien, como nos recuerda el gran gurú de la creatividad 
a debate Preexistencias en la ciudad histórica contemporánea: viabilidad y convivencia

| coordinan Plácido González Martínez, Miguel Ángel Tabales Rodríguez

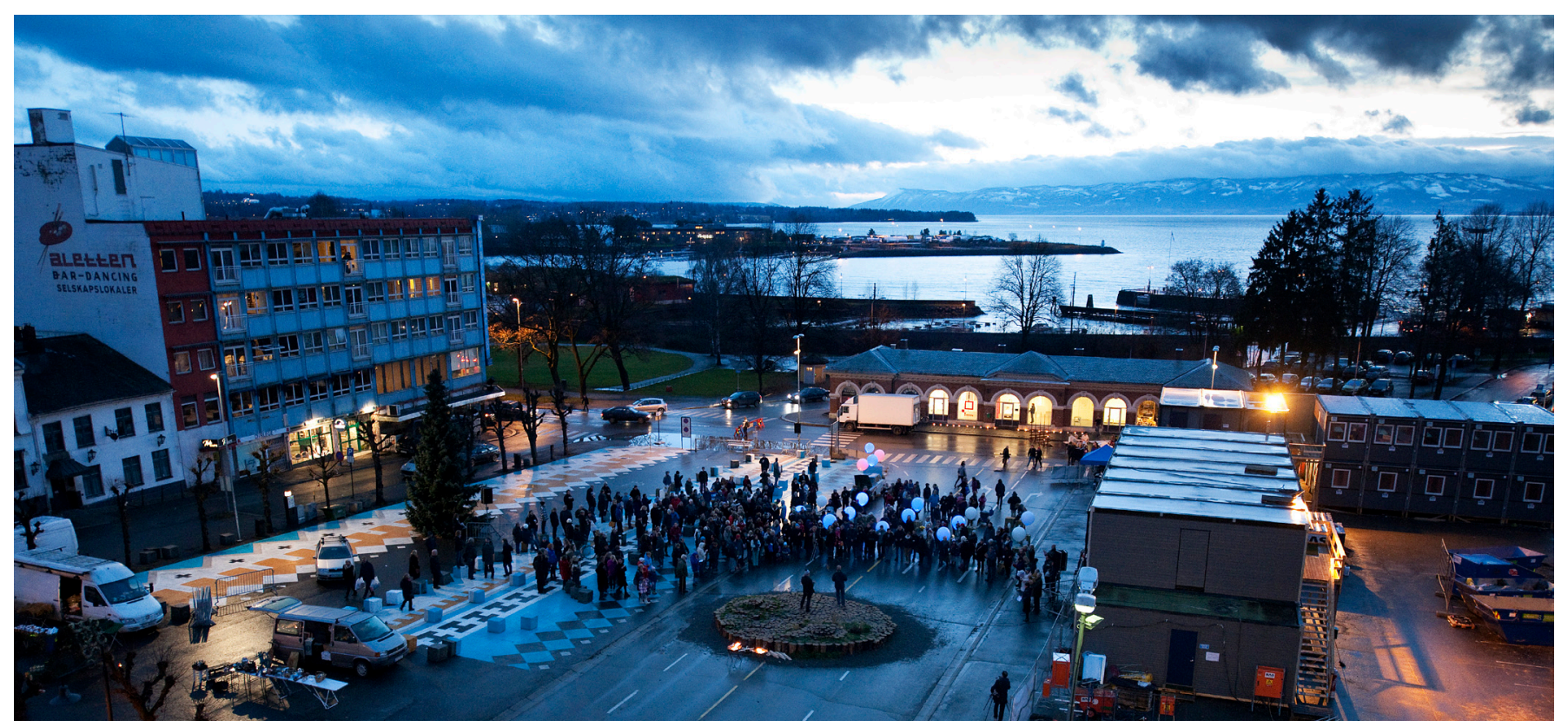

Dreamhamar, Hamar, Noruega (Arquitectos: Ecosistema Urbano, 2011-2013)

| foto Christoffer Horsfjord Nilsen/Ecosistema Urbano - CC BY-SA (https://www.flickr.com/photos/dreamhamar/)

urbana, Nabeel Hamdi, nos encontramos con la paradoja de que los países en vías de desarrollo ofrezcan más facilidades para la participación que nuestras avanzadas, democráticas y muy reguladas sociedades occidentales contemporáneas (HAMDI, 2004).

\section{Conclusión}

Esta atención a factores cada vez más complejos ha de inscribirse dentro de una apuesta verdadera por hacer que una visión política del patrimonio urbano trascienda a la intervención contemporánea en los centros históricos. En ella, un nuevo paradigma estético, una nueva belleza, llega como expresión de actores hasta ahora acallados, como era el caso de la población residente, o de la propia fauna y la flora, recuperando la voz de la naturaleza y el territorio como primeras y legítimas preexistencias.

De esta manera se establecen distancias respecto a una contemplación exclusivamente mercantilista sobre los centros históricos, en los cuales las preexistencias frecuentemente son relegadas a la condición de escenarios, "marcos incomparables" que acompañen el flujo constante de turistas, celebrantes y forasteros. Actualizando las enseñanzas de la Tendenza, el paisaje histórico y la creatividad urbana se ofrecen como herramientas útiles para reclamar el derecho a la ciudad que nos recordaba Henri Lefebvre desde los 70. Un derecho que solamente a través de la atención y del cuidado podrá garantizarse en libertad.

\section{NOTAS}

1. Un interesante estudio al efecto fue publicado como catálogo de una exposición celebrada en la Arquería de Nuevos Ministerios en Madrid, tratando los casos de Barcelona, Bilbao, Granada, Murcia, Valencia y Zaragoza (SÁNCHEZ LAMPREAVE, 2010).

2. La visión de Saverio Muratori sobre una "historia operativa" de la arquitectura sirvió un fundamento sólido al desarrollo de una teoría de 
la intervención en centros históricos que tuvo especial predicamento en Italia. Una síntesis sobre su aportación puede encontrarse en CATALDI; MAFFEI; VACCARO, 2002.

3. La visión culturalista fue desarrollada a mediados de los años 60 por Françoise Choay en L'urbanisme. Utopies et réalités (1965) y, más recientemente, enriquecida y actualizada por Carlos García Vázquez en Ciudad hojaldre. Visiones urbanas del siglo XXI (2004).

4. Una reflexión interesante sobre el paralelismo de las figuras de Lefebvre y Gramsci y sus aportaciones acerca de las dinámicas entre centros históricos y periferias pueden encontrarse en KIPFER, 2002.

5. Impulsada por la UNESCO desde 2005, la iniciativa sobre la conservación del paisaje histórico urbano recibe atención creciente, de manera especial en las ciudades de patrimonio mundial. Entre las numerosas publicaciones de referencia, resulta de interés consultar VAN OERS; HARAGUCHI, 2010.

6. Los estudios sobre creatividad urbana fueron iniciados en los años 70 por Charles Landry al frente de la empresa COMEDIA. Entre sus publicaciones de referencia, véase LANDRY, 2012.

7. Como demostró la conferencia Minding the Gap (Getty Conservation Institute, Los Angeles, 2013), buena parte de los discursos siguen gravitando en torno a conceptos conocidos como la analogía y el contraste, con los cuales difícilmente pueden sostenerse respuestas sensibles a la complejidad inherente a la ciudad contemporánea. <http:// www.getty.edu/conservation/publications resources/public_programs/ conferences/minding_gap_sympos.html> [Consulta: 17/01/2015].

8. Un interesante estudio sobre las implicaciones de este proceso puede encontrarse en STIMMANN, 2000.

9. Una completísima descripción de las intervenciones que actualmente se desarrollan en las riberas de la Saône puede encontrarse en la web <http://www.lesrivesdesaone.com> [Consulta: 01/02/2015]

10. Convertido en el más mediático autor sobre ciudades creativas desde inicios de siglo, las teorías de Richard Florida han encontrado una contestación cada vez mayor por parte de quienes ven aparejados riesgos evidentes en su asunción incondicional por parte de los poderes públicos. Se recomienda la lectura de la obra de referencia FLORIDA, 2005. Entre aquellos opuestos a las teorías de Florida, es interesante el análisis de KRÄTKE, 2010.

11. Una síntesis interesante de estos retos puede encontrarse en FERNÁNDEZ SALINAS, 2005.

\section{BIBLIOGRAFÍA}

- CATALDI, G.; MAFFEI, G. L.; VACCARO, P. (2002) Saverio Muratori and the Italian School of planning typology. Urban Morphology, 2002, 6(1), pp. 3-14

- ClÉmENT, G. (2005) Manifesto del Terzo paessagio. Macerata: Quodlibet, 2005

- ESTÉVEZ, X. (2004) Opinión y práctica en la ciudad histórica. Quintana, n. ${ }^{\circ}$ 3, 2004, pp. 25-44

- FERNÁNDEZ SALINAS, V. (2005). De la protección a la legitimación social del patrimonio urbano en España. Scripta Nova. Revista electrónica de geografía y ciencias sociales, vol. IX, n. ${ }^{0} 194$ (41) <http://www.ub.es/geocrit/sn/sn-194-41.htm>

- FlORIDA, R. (2005) Cities and the Creative Class. New York: Routledge, 2005

- HALBWACHS, M. (1995) Memoria colectiva y memoria histórica. Revista REIS, 69/95, pp. 209-219

- HAMDI, N. (2004). Small Change. About the Art of Practice and the Limits of Planning in Cities. London: Earthscan, 2004

- KIPFER, S. (2002) Urbanization, everyday life and the survival of capitalism: Lefebvre, Gramsci and political hegemony. Capitalism nature socialism, 13:2, 2002, pp. 117-149

- KRÄTKE, S. (2010) Creative cities and the rise of the dealer class: a critique of Richard Florida's approach to urban theory. International Journal of Urban and Regional Research, vol. 34.4, December 2010, pp. 835-853

- LANDRY, C. (2012) The Creative City. A Toolkiy for Urban Innovators. London: Earthscan, 2012

- PANERO PARDO, Á. (2011) Patrimonio mundial y modelo de ciudad. Ciudadanos, energía y medio ambiente en Santiago de Compostela. En FERNÁNDEZ-BACA CASARES, R.; SALMERÓN ESCOBAR, P.; SANZ, N. (ed.). El paisaje histórico urbano en las Ciudades Patrimonio Mundial. Indicadores para su conservación y gestión II. Sevilla: Consejería de Cultura de la Junta de Andalucía, 2011, pp. 227-237

- ROSSI, A. (1999) La arquitectura de la ciudad. Barcelona: Gustavo Gili, 1999

- SÁNCHEZ LAMPREAVE, R. (ed.) (2010) Las otras grandes vías. Miradas a su tiempo. Madrid: Ministerio de Vivienda: Lampreave, 2010

- StimmanN, H. (ed.) (2000) Berlin 1940-1953-1989-20002010. Physiognomie einer Grossstadt. Milano: Skira Editore, 2000

- VAN OERS, R.; HARAGUCHI, S. (ed.) (2010) Managing Historic Cities / Gérer les villes historiques. UNESCO World Heritage Papers, n. ${ }^{\circ} 27$, Septiembre $2010<$ <ttp://whc.unesco. org/en/series/27> [Consulta: 28/01/2015] 